\title{
Evaluation of optical coherence tomography angiography parameters in patients treated with Hydroxychloroquine
}

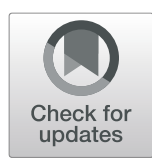

Mohammadreza Akhlaghi, Farzan Kianersi, Hamed Radmehr*, Alireza Dehghani, Afsaneh Naderi Beni and

Pegah Noorshargh

\begin{abstract}
Background: One of the major side effects of Hydroxychloroquine (HCQ) is retinopathy. The aim of this study was to evaluate the Optical coherence tomography angiography (OCTA) parameters in a group of patients who have Hydroxychloroquine-induced retinopathy based on Multifocal electroretinography (mfERG) with a group who do not have retinopathy.

Method: This is a Cross-Sectional Study. In this study, patients with Rheumatoid arthritis (RA) or Systemic lupus erythematosus (SLE) who had been taking Hydroxychloroquine for at least 7 years were included. MfERG and OCTA imaging were performed for all patients. Patients were divided into Normal mfERG and Abnormal mfERG groups based on mfERG results. OCTA parameters were studied in these two groups.

Result: Sixty-one patients (61 eyes) were included. Forty-one patients had SLE and 20 patients had RA. Forty patients (66.7\%) had Abnormal mfERG. The mean vascular density (VD) in Superficial capillary plexus (SCP) layer was not significantly different between Normal mfERG and Abnormal mfERG groups (P-Value> 0.05). Mean VD in SCP layer was not significantly different between Normal mfERG and Abnormal mfERG groups (P-Value> 0.05). In RA subgroup, mean VD in SCP layer in PeriFovea region in Abnormal mfERG group was significantly lower than normal group ( $P$-Value $<0.05)$. Mean VD in deep capillary plexus (DCP) layer in Whole Image, Superior Hemi, Inferior Hemi, PeriFovea area in Abnormal mfERG group was significantly lower than normal group ( $P$-Value $<0.05)$. This discrepancy was also observed in the RA subgroup but not in the SLE subgroup. The mean of none of the parameters of foveal avascular zone (FAZ) (mm2), Flow Area of Outer Retina (mm2) and Flow Area of Choriocapillaris (mm2) were not statistically significant between the groups Abnormal mfERG and Normal mfERG. (p-value> 0.05).

Conclusion: VD in the DCP layer decreased in abnormal mfERG patients compared to patients with normal mfERG. But it seems that VD in SCP layer, FAZ Area and Flow Area are similar in both groups. OCTA may be used as a noninvasive tool in the diagnosis of early stages of HCQ-induced retinopathy, especially in RA patients, but further studies are needed.
\end{abstract}

Keywords: Optical coherence tomography angiography, Hydroxychloroquine, Vessel density, Foveal avascular zone

\footnotetext{
* Correspondence: hkh2646@gmail.com

Isfahan Eye Research Center, Department of Ophthalmology, Isfahan

University of Medical Science, Isfahan, Iran
}

(c) The Author(s). 2021 Open Access This article is licensed under a Creative Commons Attribution 4.0 International License, which permits use, sharing, adaptation, distribution and reproduction in any medium or format, as long as you give appropriate credit to the original author(s) and the source, provide a link to the Creative Commons licence, and indicate if changes were made. The images or other third party material in this article are included in the article's Creative Commons licence, unless indicated otherwise in a credit line to the material. If material is not included in the article's Creative Commons licence and your intended use is not permitted by statutory regulation or exceeds the permitted use, you will need to obtain permission directly from the copyright holder. To view a copy of this licence, visit http://creativecommons.org/licenses/by/4.0/ The Creative Commons Public Domain Dedication waiver (http://creativecommons.org/publicdomain/zero/1.0/) applies to the data made available in this article, unless otherwise stated in a credit line to the data. 


\section{Background}

Hydroxychloroquine (HCQ) is an anti-malarial drug that is widely used in the treatment of rheumatic diseases including Systemic lupus erythematosus (SLE) and Rheumatoid arthritis (RA) [1]. An important ocular complication of HCQ is retinal toxicity [2-4], the mechanism of which is not well understood. HCQ is likely to bind to melanin within retinal pigment epithelium (RPE) cells, and its concentration in RPE cells increases over time and disrupts retinal cell metabolism and destroys photoreceptors and the Outer Nuclear Layer (ONL) [5-7].

HCQ-induced retinopathy is irreversible and can cause blindness, and may persist even after discontinuation of the drug $[8,9]$. And if toxicity is detected before RPE cell damage occurs, it can maintain the patient's central vision [10]. According to previous studies, the prevalence of this complication at doses less than $5 \mathrm{mg} / \mathrm{kg} /$ daily based on real body weight in the first 10 years of drug use is less than 2\% [11-13]. However, in more than 10 years of use of the drug and the presence of kidney disease or concomitant use of tamoxifen, it reaches 20\% [14].

To screen patients for HCQ-induced retinopathy as recommended by the American Association of Ophthalmology (AAO), all patients are screened at the start of medication, then annually after 5 years of medication, and individuals Which have a major Risk Factor are examined at shorter intervals. To diagnose HCQ-induced retinopathy, various methods have been recommended, including: Spectral Domain Optical Coherence Tomography (SD-OCT), automated perimetry test, multifocal electroretinogram (mfERG), FUNDUS AUTOFLUORESCENCE (FAF) IMAGING [15]. But it is controversial as to which of these methods is the Gold Standard early detection of HCQ-induced retinopathy [15-20]. So it's important to find a tool that can help us diagnose early.

mfERG is highly sensitive among the mentioned tests and because it is an objective test, it is less dependent on the patient's response and cooperation [15]. The mfERG objectively evaluates the electroretinographic response of the macular region, and in HCQ retinopathy, this response is reduced in the paracentral region [17].

Optical coherence tomography angiography (OCTA) is a new imaging technique without contrast injection that can show the vascular structure of the retina and choroid based on the flow of red blood cells and It is used to study the course and pathogenesis of retinal diseases such as Diabetic retinopathy, Choroidal neovascularization (CNV), Central retinal vein occlusion (CRVO) [21]. It may also be helpful in the early detection of HCQ-induced retinopathy. To the best of our knowledge, there are very few articles in this field [22, 23].

The aim of our study was to compare the quantitative and qualitative parameters of OCTA including vascular density, foveal avascular zone (FAZ) with MFERG changes in patients with a history of HCQ. We think this study may help to better understand the mechanism of HCQ-induced retinopathy and its early diagnosis.

\section{Method}

This is a cross-sectional study performed in retina clinic of Feiz Hospital and Didavaran Eye Center, Isfahan, Iran in 2019-2020, Approved by the Clinical Research Ethics Committee of Isfahan University of Medical Sciences. Informed consent was obtained from all patients participating in the study.

All patients with a history of at least 7 years of Hydroxychloroquine due to Systemic lupus erythematosus (SLE) or rheumatoid arthritis (RA), who referred to the retinal clinic of Feiz Hospital during 2019-2020 were included in this study. One eye from each patient was included in the study, so that if the patient's month of birth was even, the right eye and if the patient's month of birth was odd, the patient's left eye was included in the study. Patients with exclusion criteria were excluded from the study, including history of ocular surgery, history of ocular disease, comorbid systemic diseases (such as hypertension (HTN) and diabetes mellitus (DM) and vascular diseases), refractive error spherical equivalent greater than 4.00D, Intraocular pressure (IOP) above 21 $\mathrm{mmHg}$, kidney disease or failure, concomitant use of tamoxifen and presence of maculopathy due to other diseases.

A complete medical and ophthalmological history was taken from all patients, including: demographic information, type of disease, duration of disease, drugs and their amount and duration of use. All patients underwent a complete ophthalmologic examination by a vitreo retinal fellowship, Includes: refraction, best corrected visual acuity (BCVA) (in decimal and LogMAR chart (Logarithm of the Minimum Angle of Resolution)), IOP, anterior segment examination and fundoscopy with dilated pupil. Spectral-domain OCT (SD-OCT), Optical coherence tomography angiography (OCT-A), multifocal electroretinogram (mfERG), Humphrey Visual Field Test 24-2, Fundus autofluorescence (FAF) were performed for all patients.

mfERG was performed by RETI Scan (Roland consulting company) and software 6.16.3.10 according to the protocol of the International Society for Clinical Electrophysiology of Vision (ISCEV) by an expert optometrist. The 61-hexagon strategy was used and the stimulus was calibrated according to the ISCEV guidelines. The mean amplitudes of N1, P1, N2 were analyzed in five concentric areas of the macula $[24,25]$.

To interpret mfERG in HCQ retinopathy screening, different criteria have been used in various articles, such as: amplitude reduction, implicit time extension, ring ratio and amplitude reduction based on Color Difference 
Plot [8, 25-27]. In this study, we considered abnormal values of R1 or R2 amplitude or Ring Ratio (R1 / R2, R1 / R3, R1 / R4, R1 / R5) in each of the patient's eyes as abnormal mfERG. The normal range of mfERG parameters was determined based on the normal database of our center, which has been corrected based on age, sex.

OCTA imaging was performed with RTVue XR device (OPTOVUE, California, USA) and software version 2018.0.0.14. Macular imaging was performed with a 6 * $6 \mathrm{~mm}$ scans. The segmentation was done automatically and checked by an expert technician. All images were taken with dilated pupil and by the same machine, and in similar conditions at 8 A.M by an expert technician. And quantitative values of vascular density (in superficial retinal capillary plexus (SCP) and deep capillary plexus (DCP)), foveal avascular zone (FAZ), Flow Rate (in choriocapillaris, outer retina layers) and Retinal Thickness automatically by the software was calculated. Images with signal strength index (SSI) less than 40 or had an artifact were not analyzed [28]. Finally, based on mfERG criteria, we divided patients into normal mfERG and abnormal mfERG groups. We also divided patients into separate SLE and RA subgroups.

\section{Statistical analysis}

Data were analyzed using the SPSS version 21.0 Statistical package (SPSS Inc., Chicago, IL, USA). Quantitative and qualitative data were presented as Mean $\pm \mathrm{SD}$, Median [maximum, minimum] and frequency (percentage). Normality of continuous data was evaluated using Kolmogorov-Smirnov test. Independent t-test was used for comparing data between groups. Qualitative data were compared between groups using chi-square test and fisher exact test. Pearson correlation were used to investigate the correlations between duration of the treatment, cumulative dose and Vessel Density, FAZ Area, Flow Area based. Statistical significance level was set at $P<0.05$.

\section{Result}

Sixty-one patients (61 eyes) with a history of at least 7 years of Hydroxychloroquine use due to SLE or RA were studied. Of these, 57 were female (93.4\%) and 4 were male $(6.5 \%)$ with a mean age of $46.9 \pm 11.62$ years. Forty patients (40 eyes) had SLE and 21 patients (21 eyes) had RA. In general, out of 61 patients (61 eyes), 40 (65.5\%) were considered in the Abnormal mfERG group. Demographic and clinical variables of individuals are shown in Table 1. Patients did not have a statistically significant difference in any of the demographic and clinical variables including age, sex, mean daily dose of HCQ, total dose and BCVA (Best-corrected visual acuity) in normal and abnormal groups (P-VALUE $>0.05)$.

\section{SCP vessel density (\%) parameters of OCTA}

Mean SCP vessel density (\%) parameters in Abnormal mfERG and Normal mfERG groups for all patients and also separately for SLE and RA subgroups are shown in Table 2. There was no significant difference between Abnormal mfERG and Normal mfERG groups in any of the SCP vessel density parameters, although in patients with RA the mean SCP vessel density (\%) of Whole image, inferior Hemi, ParaFovea and PeriFovea in the Abnormal mfERG group were significantly lower than in the normal group $(P$-VALUE $<0.05)$.

\section{DCP vessel density (\%) parameters of OCTA}

Mean DCP vessel density (\%) parameters in Abnormal mfERG and Normal mfERG groups for all patients and also separately for SLE and RA sub groups are shown in Table 3. There was a decrease in mean DCP vessel density (\%) in the Abnormal mfERG group compared to the normal group in the areas of Whole image, Superior Hemi, Inferior Hemi, PeriFove, which is statistically significant. (P-VALUE $<0.05)$. This difference is also observed in the subgroup of people with RA, but no significant difference was observed in the SLE subgroup.

\section{FAZ and FLOW area parameters of OCTA}

Mean FAZ $\left(\mathrm{mm}^{2}\right)$ and FLOW Area $\left(\mathrm{mm}^{2}\right)$ Parameters in Abnormal mfERG and Normal mfERG groups for all patients and also separately for SLE and RA subgroups are shown in Table 3. The mean of none of the parameters of FAZ $\left(\mathrm{mm}^{2}\right)$, Flow Area of Outer Retina $\left(\mathrm{mm}^{2}\right)$ and Flow Area of Choriocapillaris $\left(\mathrm{mm}^{2}\right)$ were not statistically significant between the groups Abnormal mfERG and Normal mfERG. ( $p$-value>0.05). However, the mean Flow Area of Choriocapillaris $\left(\mathrm{mm}^{2}\right)$ in RA subgroup with Abnormal mfERG was statistically significantly lower than RA subgroup with Normal mfERG ( $p$-value $<0.05)$.

Table 4 shows the relationship between Vessel Density, Retinal Thickness, FAZ, and Flow Area with the cumulative 1 dose of Hydroxychloroquine and the duration of treatment. The mean SCP Vessel Density in Whole Image and PeriFovea regions, as well as the mean DCP Vessel Density in Whole Image and PeriFovea regions showed a strong and inverse relationship with duration of treatment and cumulative dose, but the mean FAZ and Flow Area in Outer Retina and Choriocapillaris did not show a statistically significant relationship with drug duration or cumulative dose of drug.

\section{Discussion}

Since early detection of HCQ-induced retinopathy is important, it is of great interest to find a modality that helps diagnose early-stage of retinopathy. In 2016, the AAO guideline proposed various modalities, including SD-OCT, AVF, FAF, and mfERG, for screening of HCQ- 
Table 1 The Demographic and Clinical characteristics of the patients

\begin{tabular}{|c|c|c|c|}
\hline & Normal mfERG $(n=21)$ & Abnormal mfERG $(n=40)$ & $P$-Value \\
\hline Sex & & & $0.289^{\circ}$ \\
\hline Female & $21(36.8)$ & $36(63.2)$ & \\
\hline Male & $0(0.0)$ & $4(100.0)$ & \\
\hline Disease $\mathrm{f}(\%)$ & & & $0.486^{\circ}$ \\
\hline SLE & $15(37.5)$ & $25(62.5)$ & \\
\hline $\mathrm{RA}$ & $6(28.6)$ & $15(71.4)$ & \\
\hline Age & & & $0.711^{\natural}$ \\
\hline Mean \pm SD & $47.0 \pm 11.94$ & $46.7 \pm 11.27$ & \\
\hline Median [min, max] & $48[23,65]$ & $46[29,62]$ & \\
\hline Duration of Treatment (years) & & & $0.813^{\natural}$ \\
\hline Mean \pm SD & $13.23 \pm 5.83$ & $13.15 \pm 5.63$ & \\
\hline Median [min, max] & $13[7,32]$ & $11.5[7,24]$ & \\
\hline Daily Dose (mg/day) & & & $0.850^{\natural}$ \\
\hline Mean \pm SD & $202.50 \pm 49.29$ & $215.0 \pm 65.09$ & \\
\hline Median [min, max] & $200[150,400]$ & $200[150,400]$ & \\
\hline Total Dose of HCQ (mg) & & & $0.418^{\natural}$ \\
\hline Mean \pm SD & $978.20 \pm 523.20$ & $1034.77 \pm 590.09$ & \\
\hline Median [min, max] & $930.75[511,2920]$ & $590.09[511,2920]$ & \\
\hline $\begin{array}{l}\text { BCVA (Best-corrected visual acuity) } \\
\text { Decimal }\end{array}$ & & & $0.782^{\natural}$ \\
\hline Mean \pm SD & $0.88 \pm 0.18$ & $0.81 \pm 0.27$ & \\
\hline Median [min, max] & $0.90[0.2,1.0]$ & $1.0[0.2,1.0]$ & \\
\hline log MAR [Logarithm of the Minimum Angle of Resolution] & & & $0.172^{9}$ \\
\hline Mean \pm SD & $0.069 \pm 0.13$ & $0.130 \pm 0.20$ & \\
\hline Median [min, max] & $0.046[0.0,0.07]$ & $0.0[0.0,0.07]$ & \\
\hline
\end{tabular}

ף: Resulted from independent t-test and $P$ value $<0.05$

(c): Resulted from chi-square test

๑: Resulted from Fisher's exact test

SLE Systemic lupus erythematosus, RA Rheumatoid arthritis, HCQ Hydroxychloroquine, mfERG Multifocal electroretinography

induced retinopathy, but none are gold standard [15]. The mfERG is more sensitive for diagnosis of HCQinduced retinopathy in the early stages than the combination of AVF and SD-OCT tests [25, 29]. For this reason, in this study, based on mfERG changes, we divided patients into two groups: patients with Abnormal mfERG (Case group) and patients with Normal mfERG (Control group) and evaluated them with OCTA imaging. Patients with RA and SLE were also evaluated in separate subgroups. Because HCQ use for more than 5 years is a high risk for HCQ-induced retinopathy [15], we enrolled patients with at least 7 years of HCQ use.

Based on our findings, the mean Vessel Density (VD) in the SCP layer was not significantly different between the Abnormal mfERG and Normal mfERG groups, although in the RA subgroup, VD in Whole Image, ParaFovea and PeriFovea of the SCP layer in the Abnormal mfERG group decreased compared to the normal group.
But it was not observed in SLE patients. The mean VD in the DCP layer (Whole Image, PeriFovea,) in the Abnormal mfERG group was lower than the normal group. This difference was also present in the RA subgroup but was not observed in the SLE subgroup. The mean VD in the Fovea area did not differ between the two groups. Also, FAZ Area and Flow Area (outer retina, choriocapillaris) were not different in the two groups and only Choriocapillaris Flow Area was lower in RA patients with Abnormal mfERG compared to RA patients with Normal mfERG.

To the best of our knowledge, limited studies have been conducted on the use of OCTA in the evaluation of HCQ-induced retinopathy, the results of which are also controversial. Some studies have evaluated OCTA changes between healthy individuals and patients with a history of HCQ use, and some studies have evaluated OCTA changes between High Risk (HCQ users over 5 
Table 2 SCP vessel density (\%) parameters of OCTA

\begin{tabular}{|c|c|c|c|c|}
\hline Variables & & SLE $(n=40)$ & $\mathrm{RA}(n=21)$ & All patients $(n=61)$ \\
\hline \multirow[t]{3}{*}{ Whole image } & Normal mfERG & $44.53 \pm 4.84$ & $50.68 \pm 2.63$ & $46.28 \pm 5.08$ \\
\hline & Abnormal mfERG & $45.28 \pm 4.60$ & $44.71 \pm 4.91$ & $45.06 \pm 4.66$ \\
\hline & $P$-Value $\odot$ & 0.627 & $0.019^{*}$ & 0.575 \\
\hline \multirow[t]{3}{*}{ Superior Hemi } & Normal mfERG & $44.75 \pm 4.72$ & $50.00 \pm 2.86$ & $46.25 \pm 4.80$ \\
\hline & Abnormal mfERG & $44.81 \pm 4.68$ & $45.00 \pm 5.81$ & $44.88 \pm 5.06$ \\
\hline & $P$-Value $\odot$ & 0.972 & 0.085 & 0.549 \\
\hline \multirow[t]{3}{*}{ Inferior Hemi } & Normal mfERG & $44.34 \pm 5.04$ & $51.22 \pm 2.43$ & $46.31 \pm 5.66$ \\
\hline & Abnormal mfERG & $45.71 \pm 4.58$ & $45.21 \pm 5.52$ & $45.52 \pm 4.88$ \\
\hline & $P$-Value $\odot$ & 0.397 & $0.032^{*}$ & 0.520 \\
\hline \multirow[t]{3}{*}{ Fovea } & Normal mfERG & $13.27 \pm 8.87$ & $17.06 \pm 3.61$ & $14.35 \pm 7.97$ \\
\hline & Abnormal mfERG & $13.66 \pm 6.83$ & $12.25 \pm 8.36$ & $13.13 \pm 7.36$ \\
\hline & $P$-Value $\odot$ & 0.876 & 0.234 & 0.549 \\
\hline \multirow[t]{3}{*}{ ParaFovea } & Normal mfERG & $43.78 \pm 7.95$ & $51.38 \pm 4.21$ & $45.95 \pm 7.30$ \\
\hline & Abnormal mfERG & $46.01 \pm 6.67$ & $42.39 \pm 6.72$ & $44.76 \pm 6.65$ \\
\hline & $P$-Value $\odot$ & 0.277 & $0.012^{*}$ & 0.626 \\
\hline \multirow[t]{3}{*}{ PeriFovea } & Normal mfERG & $45.69 \pm 5.45$ & $51.98 \pm 2.75$ & $47.49 \pm 5.54$ \\
\hline & Abnormal mfERG & $46.01 \pm 4.52$ & $46.41 \pm 4.98$ & $46.15 \pm 4.62$ \\
\hline & $P$-Value $\odot$ & 0.845 & $0.030^{*}$ & 0.324 \\
\hline
\end{tabular}

${ }^{*} P$ value $<0.05$ and analysis using by independent t-test

SLE Systemic lupus erythematosus, RA Rheumatoid arthritis, mfERG Multifocal electroretinography, OCTA Optical coherence tomography angiography, SCP Superficial capillary plexus

years) and Low Risk (HCQ users less than 5 years) [22, 23, 28, 30-32]. A review of studies in this field shows confusing results. To our knowledge, no study has been performed to evaluate changes in OCTA among patients with HCQ-induced retinopathy based on MfERG and those without retinopathy.

In the study of Tarakcioglu et al., which was performed among 102 patients, No significant difference was observed between the HCQ treatment group and the control group in the parameters of FAZ, Flow Area, vascular density, SCP and DCP [28]. Based on our results, the patients in both groups were similar in the parameters of FAZ, Flow Area and SCP vascular density. In Bulut et al. study, the mean of vascular density (both SCP and DCP layers) in the group with HCQ use $<5$ years was higher than the group with $\mathrm{HCQ}$ use $>5$ years and also the FAZ Area in the group with HCQ use $>5$ years was wider [22]; While in our study, vascular density in DCP was significantly different between the two groups, Their study was performed in patients with RA, Sjogren, SLE and Connective tissue disease, While our study was performed in SLE and RA patients; The duration of treatment and the cumulative dose of HCQ were also lower than in our study. In Goker et al., The mean VD of the HCQ group was lower than group of healthy individuals, and the FAZ Area was higher in the
HCQ group than in healthy individuals [30]. In this study, patients were compared with healthy people. Since rheumatologic diseases, especially SLE, affect retinal vascularity [33], the results may not be related to HCQ. While we compared OCTA parameters in two groups of patients that can eliminate vascular changes caused by the disease itself.

In the Forte et al. study, was reported that with increasing cumulative dose and duration of HCQ use, VD in SCP and DCP decreased and also FAZ Area increased with Swept-source optical coherence tomography (SSOCT) and separately, the vascular density in the areas of Fovea, PeriFovea, ParaFovea was not determined and the number of patients was 10 [23]. Based on our results with RTVue XR-OCTA, VD, especially in the PeriFovea area, is inversely related to the cumulative dose and duration of $\mathrm{HCQ}$.

In our study, changes in vascular density, especially in the DCP layer in RA patients, differed between the two groups of Abnormal mfERG and Normal mfERG. The Telek et al. study also found that HCQ-induced retinopathy changes were more common in RA patients than in SLE patients. This may be due to longer use of HCQ or older patients [34]. Ozek et al. evaluated changes in OCTA in RA patients. VD in the DCP layer (Deep Temporal, Deep Hemi inferior) was lower in RA patients 
Table 3 DCP vessel density (\%) parameters of OCTA and FAZ And Flow Area

\begin{tabular}{|c|c|c|c|c|}
\hline Variables & & SLE $(n=40)$ & RA $(n=21)$ & All patients $(n=61)$ \\
\hline \multirow[t]{3}{*}{ Whole image } & Normal mfERG & $42.71 \pm 10.59$ & $53.76 \pm 6.10$ & $45.86 \pm 10.60$ \\
\hline & Abnormal mfERG & $39.99 \pm 7.46$ & $40.44 \pm 9.12$ & $40.16 \pm 8.17$ \\
\hline & $P$-Value $\odot$ & 0.347 & $0.009^{*}$ & $0.023^{*}$ \\
\hline \multirow[t]{3}{*}{ Superior Hemi } & Normal mfERG & $42.31 \pm 10.36$ & $52.9 \pm 5.36$ & $45.33 \pm 10.41$ \\
\hline & Abnormal mfERG & $39.81 \pm 7.61$ & $40.64 \pm 9.11$ & $40.12 \pm 8.10$ \\
\hline & P-Value $\odot$ & 0.387 & $0.012^{*}$ & $0.034^{*}$ \\
\hline \multirow[t]{3}{*}{ Inferior Hemi } & Normal mfERG & $43.07 \pm 11.21$ & $54.72 \pm 6.71$ & $46.40 \pm 11.35$ \\
\hline & Abnormal mfERG & $40.16 \pm 7.67$ & $40.26 \pm 10.30$ & $40.20 \pm 8.62$ \\
\hline & P-Value $\odot$ & 0.335 & $0.009^{*}$ & $0.020^{*}$ \\
\hline \multirow[t]{3}{*}{ Fovea } & Normal mfERG & $29.67 \pm 8.87$ & $35.7 \pm 5.87$ & $31.39 \pm 8.25$ \\
\hline & Abnormal mfERG & $30.61 \pm 9.52$ & $26.49 \pm 12.52$ & $29.07 \pm 10.79$ \\
\hline & $P$-Value $\odot$ & 0.7590 & 0.133 & 0.447 \\
\hline \multirow[t]{3}{*}{ ParaFovea } & Normal mfERG & $49.25 \pm 9.92$ & $57.96 \pm 2.94$ & $51.74 \pm 9.32$ \\
\hline & Abnormal mfERG & $49.56 \pm 6.70$ & $46.24 \pm 9.70$ & $48.32 \pm 8.01$ \\
\hline & $P$-Value $\odot$ & 0.906 & $0.018^{*}$ & 0.187 \\
\hline \multirow[t]{3}{*}{ PeriFovea } & Normal mfERG & $42.71 \pm 12.10$ & $55.94 \pm 6.60$ & $46.44 \pm 12.32$ \\
\hline & Abnormal mfERG & $39.88 \pm 8.48$ & $41.14 \pm 10.3$ & $40.36 \pm 9.09$ \\
\hline & $P$-Value $\odot$ & 0.391 & $0.008^{*}$ & $0.031^{*}$ \\
\hline \multirow[t]{3}{*}{ FAZ (.mm2) } & Normal mfERG & $0.38 \pm 0.41$ & $0.27 \pm 0.05$ & $0.35 \pm 0.35$ \\
\hline & Abnormal mfERG & $0.36 \pm 0.18$ & $0.31 \pm 0.18$ & $0.34 \pm 0.18$ \\
\hline & $P$-Value $\odot$ & 0.784 & 0.748 & 0.899 \\
\hline \multirow[t]{3}{*}{ Flow Area of Outer Retina $\left(\mathrm{mm}^{2}\right)$} & Normal mfERG & $1.40 \pm 0.58$ & $0.93 \pm 0.52$ & $1.27 \pm 0.59$ \\
\hline & Abnormal mfERG & $1.17 \pm 0.56$ & $1.44 \pm 0.46$ & $1.27 \pm 0.53$ \\
\hline & P-Value $\odot$ & 0.235 & 0.060 & 0.944 \\
\hline \multirow[t]{3}{*}{ Flow Area of Choriocapillaris $\left(\mathrm{mm}^{2}\right.$ ) } & Normal mfERG & $2.16 \pm 0.11$ & $2.27 \pm 0.06$ & $2.19 \pm 0.11$ \\
\hline & Abnormal mfERG & $2.15 \pm 0.12$ & $2.08 \pm 0.17$ & $2.12 \pm 0.15$ \\
\hline & $P$-Value $\odot$ & 0.693 & $0.002^{*}$ & $0.050^{*}$ \\
\hline
\end{tabular}

${ }^{*} P$ value $<0.05$ and analysis using by independent $\mathrm{t}$-test

SLE Systemic lupus erythematosus, RA Rheumatoid arthritis, mfERG Multifocal electroretinography, OCTA Optical coherence tomography angiography, DCP Deep capillary plexus, FAZ Foveal avascular zone

with a history of HCQ of more than 5 years compared to healthy individuals and also compared to individuals with a history of HCQ of less than 5 years [31].

In our study, we concluded that VD in the SCP layer (Whole Image, PeriFovea) was inversely related to the cumulative dose of the drug and the duration of drug administration. Also, VD in the DCP layer (Whole Image, PeriFovea) is inversely proportional to the duration of HCQ consumption. However, no relationship was observed between FAZ Area and Flow Area with drug duration and cumulative dose of HCQ. The mechanism of HCQ-induced retinal toxicity is not well understood. According to some studies, the accumulation of HCQ in RPE cells interferes with the clearance of intracellular toxic substances. That the inner layers and outer layers of the retina are damaged and some studies have suggested that the inner layer of the retina is not involved [6]. In the study of Kan et al. stated that ganglion cell-inner plexiform layer (GC-IPL) thinning occurs in patients with a history of HCQ before changes in AVF and RPE changes [35]. The study by Bulut et al. also showed that in the early stages of $\mathrm{HCQ}$-induced retinal toxicity, the thickness of GC-IPL decreases. This reduction is related to the cumulative dose and duration of HCQ use [36]. In our study, DCP changes were greater than SCP, although these changes were not observed in SLE patients. In the study of De Sisternes et al., it was stated that HCQ retinopathy first begins in the photoreceptors and the paraFovea region, and that the Fovea is involved in more advanced stages [5]. In our study, FAZ Area and VD in Fovea Area in the two groups were not significantly different, which could be due to the fact that Fovea area is not involved in the early and subclinical stages of HCQ retinopathy. Flow 
Table 4 Pearson correlation between Duration of the treatment, Cumulative dose and Vessel Density, FAZ, Flow Area based

\begin{tabular}{|c|c|c|}
\hline Variables & Duration of treatment (p) & Cumulative dose $(p)$ \\
\hline \multicolumn{3}{|l|}{ SCP vessel Density (\%) } \\
\hline Whole Image & $-0.356(0.005)^{*}$ & $-0.294(0.021)^{*}$ \\
\hline Fovea & $0.064(0.622)$ & $-0.008(0.952)$ \\
\hline ParaFovea & $-0.236(0.067)$ & $-0.213(0.099)$ \\
\hline PeriFovea & $-0.403(0.001)^{*}$ & $-0.330(0.009)^{*}$ \\
\hline \multicolumn{3}{|l|}{ DCP Vessel Density (\%) } \\
\hline Whole Image & $-0.272(0.034)^{*}$ & $-0.278(0.030)^{*}$ \\
\hline Fovea & $0.094(0.469)$ & $-0.21(0.869)$ \\
\hline ParaFovea & $-0.177(0.177)$ & $-0.181(0.163)$ \\
\hline PeriFovea & $-0.272(0.034)^{*}$ & $-0.274(0.032)^{*}$ \\
\hline $\mathrm{FAZ}\left(\mathrm{mm}^{2}\right)$ & $-0.064(0.627)$ & $-0.057(0.664)$ \\
\hline Flow Area of Outer Retina $\left(\mathrm{mm}^{2}\right)$ & $0.188(0.147)$ & $0.098(0.451)$ \\
\hline Flow Area of Choriocapillaris ( $\left.\mathrm{mm}^{2}\right)$ & $-0.206(0.112)$ & $-0.171(0.187)$ \\
\hline
\end{tabular}

*: $P$ value $<0.05$ and analysis using by pearson correlation test

SCP Superficial capillary plexus, DCP Deep capillary plexus, FAZ Foveal avascular zone, HCQ Hydroxychloroquine

area in Choriocapillaris was lower in RA patients with abnormal mfERG than in the other group, which may indicate that Choriocapillaris is involved in early HCQ retinopathy, but this difference was not observed in the SLE group, so further studies are needed.

The patients we evaluated all had normal AVF, FAF, SD-OCT, but some had evidence of early stage of HCQinduced retinopathy at mfERG.

The strength of our study compared to other studies is that we performed OCTA in patients with a history of HCQ in two groups: Abnormal mfERG (with subclinical or early stage of HCQ Retinopathy) and Normal mfERG (without HCQ Retinopathy). To evaluate whether OCTA can help as a sensitive test to diagnose subclinical or early-stage retinopathy. Another feature of our study is that we separately evaluated RA and SLE patients who may have different immunological mechanisms. The limitations of our study are that it is cross-sectional and retrospective and the sample size is small, so doing a prospective study with more patients will help to get more accurate results. Also, the study of OCTA parameters in patients who have proven retinopathy due to HCQ in all three tests SD-OCT, mfERG, AVF can provide us with more useful information. But usually, the number of these patients is small.

In conclusion, we found that the density of retinal vessels in the Perifovea area decreased with increasing cumulative dose of and duration of treatment, but the FAZ Area did not change significantly. In RA patients with Abnormal mfERG (early stage of HCQ Retinopathy) mean VD of PeriFovea, ParaFovea area of DCP layer was reduced compared to RA patients with Normal mfERG (without HCQ Retinopathy) but FAZ Area and VD of Fovea did not change significantly in early retinopathy.
OCTA may be used as a non-invasive modality to diagnose early stages of HCQ retinopathy but our knowledge in this area is still limited and more studies are needed.

\section{Abbreviations}

SLE: Systemic lupus erythematosus; RA: Rheumatoid arthritis;

HCQ: Hydroxychloroquine; mfERG: Multifocal electroretinography;

OCTA: Optical coherence tomography angiography; SCP: Superficial capillary plexus; DCP: Deep capillary plexus; FAZ: Foveal avascular zone

\section{Acknowledgements}

Thanks to the staff of Didavaran Clinic and Feiz Hospital, especially Ms. Neda Mazaheri and Ms. Elahe Salehnia who helped us in this research.

\section{Authors' contributions}

Dr. Mohammad Reza Akhlaghi, Dr. Farzan Kianersi, Dr. Alireza Dehghani and Dr. Hamed Radmehr collected patient information and examined patients. Dr. Hamed Radmehr and Dr. Afsaneh Naderi helped write the original manuscript. Pegah Noor Shargh analyzed the data, prepared the tables, and helped write the results. All authors read and approved the manuscript.

Funding

None.

Availability of data and materials

The datasets used and/or analyzed during the current study are available from the corresponding author on reasonable request.

\section{Declarations}

\section{Ethics approval and consent to participate}

This study was performed according to Declaration of Helsinki and approved by the Institutional Research Ethics committee, School of Medicine - Isfahan University of Medical Sciences. The Approval ID is IR.MUI.MED.REC.1398.209. All participants were above 16 years of age and written Informed consent was obtained from all patients participating in the study.

Consent for publication

not applicable.

Competing interests

The authors declare that they have no competing interests. 
Received: 18 February 2021 Accepted: 4 May 2021

Published online: 11 May 2021

\section{References}

1. Rainsford K, Parke AL, Clifford-Rashotte M, Kean W. Therapy and pharmacological properties of hydroxychloroquine and chloroquine in treatment of systemic lupus erythematosus, rheumatoid arthritis and related diseases. Inflammopharmacology. 2015;23(5):231-69.

2. Geamnu A, Popa-Cherecheanu A, Marinescu B, Geamanu C, Voinea L. Retinal toxicity associated with chronic exposure to hydroxychloroquine and its ocular screening. Review. J Med Life. 2014;7(3):322.

3. Jover J, Leon L, Pato E, Loza E, Rosales Z, Matias M, et al. Long-term use of antimalarial drugs in rheumatic diseases. Clin Exp Rheumatol-Incl Supplements. 2012;30(3):380.

4. Ruiz-Irastorza G, Ramos-Casals M, Brito-Zeron P, Khamashta MA. Clinical efficacy and side effects of antimalarials in systemic lupus erythematosus: a systematic review. Ann Rheum Dis. 2010;69(01):20-8.

5. de Sisternes L, Hu J, Rubin DL, Marmor MF. Localization of damage in progressive hydroxychloroquine retinopathy on and off the drug: inner versus outer retina, parafovea versus peripheral fovea. Invest Ophthalmol Vis Sci. 2015;56(5):3415-26.

6. Lee MG, Kim SJ, Ham D-I, Kang SW, Kee C, Lee J, et al. Macular retinal ganglion cell-inner plexiform layer thickness in patients on hydroxychloroquine therapy. Invest Ophthalmol Vis Sci. 2015;56(1):396-402.

7. Marmor MF. Comparison of screening procedures in hydroxychloroquine toxicity. Arch Ophthalmol. 2012:130(4):461-9.

8. Michaelides M, Stover NB, Francis PJ, Weleber RG. Retinal toxicity associated with hydroxychloroquine and chloroquine: risk factors, screening, and progression despite cessation of therapy. Arch Ophthalmol. 2011;129(1):30-9.

9. Wei L, Chen S, Ho C, Kuo Y, Ho J. Progression of hydroxychloroquine retinopathy after discontinuation of therapy: case report. Chang Gung Med J. 2001:24(5):329-34

10. Marmor MF, Hu J. Effect of disease stage on progression of hydroxychloroquine retinopathy. JAMA Ophthalmol. 2014;132(9):1105-12.

11. Levy G, Munz S, Paschal J, Cohen H, Pince K, Peterson T. Incidence of hydroxychloroquine retinopathy in 1,207 patients in a large multicenter outpatient practice. Arthritis Rheum. 1997;40(8):1482-6.

12. Mavrikakis I, Sfikakis PP, Mavrikakis E, Rougas K, Nikolaou A, Kostopoulos C, et al. The incidence of irreversible retinal toxicity in patients treated with hydroxychloroquine: a reappraisal. Ophthalmology. 2003;110(7):1321-6.

13. Wolfe F, Marmor MF. Rates and predictors of hydroxychloroquine retinal toxicity in patients with rheumatoid arthritis and systemic lupus erythematosus. Arthritis Care Res. 2010;62(6):775-84.

14. Melles RB, Marmor MF. The risk of toxic retinopathy in patients on longterm hydroxychloroquine therapy. JAMA Ophthalmol. 2014;132(12):1453-60.

15. Marmor MF, Kellner U, Lai TY, Melles RB, Mieler WF. Recommendations on screening for chloroquine and hydroxychloroquine retinopathy (2016 revision). Ophthalmology. 2016;123(6):1386-94.

16. Kellner S, Weinitz S, Kellner U. Spectral domain optical coherence tomography detects early stages of chloroquine retinopathy similar to multifocal electroretinography, fundus autofluorescence and near-infrared autofluorescence. Br J Ophthalmol. 2009:93(11):1444-7.

17. Lai TY, Ngai JW, Chan W-M, Lam DS. Visual field and multifocal electroretinography and their correlations in patients on hydroxychloroquine therapy. Doc Ophthalmol. 2006;112(3):177-87.

18. Marmor MF, Chien FY, Johnson MW. Value of red targets and pattern deviation plots in visual field screening for hydroxychloroquine retinopathy. JAMA Ophthalmol. 2013;131(4):476-80.

19. Marmor MF, Melles RB. Disparity between visual fields and optical coherence tomography in hydroxychloroquine retinopathy. Ophthalmology. 2014;121(6):1257-62

20. Mititelu M, Wong BJ, Brenner M, Bryar PJ, Jampol LM, Fawzi AA. Progression of hydroxychloroquine toxic effects after drug therapy cessation: new evidence from multimodal imaging. JAMA ophthalmology. 2013;131(9):1187-97.

21. Pichi F, Sarraf D, Arepalli S, Lowder CY, Cunningham ET Jr, Neri $P$, et al. The application of optical coherence tomography angiography in uveitis and inflammatory eye diseases. Prog Retin Eye Res. 2017:59:178-201.

22. Bulut M, Akıdan M, Gözkaya O, Erol MK, Cengiz A, Çay HF. Optical coherence tomography angiography for screening of hydroxychloroquine-induced retinal alterations. Graefes Arch Clin Exp Ophthalmol. 2018;256(11):2075-81.
23. Forte R, Haulani H, Dyrda A, Jürgens I. Swept source optical coherence tomography angiography in patients treated with hydroxychloroquine: correlation with morphological and functional tests. Br J Ophthalmol. 2019;1:1.

24. Hood DC, Bach $M$, Brigell $M$, Keating D, Kondo M, Lyons JS, et al. ISCEV standard for clinical multifocal electroretinography (mfERG) (2011 edition). Doc Ophthalmol. 2012;124(1):1-13.

25. Tsang AC, Pirshahid SA, Virgili G, Gottlieb CC, Hamilton J, Coupland SG. Hydroxychloroquine and chloroquine retinopathy: a systematic review evaluating the multifocal electroretinogram as a screening test. Ophthalmology. 2015;122(6):1239-51.e4

26. Lyons JS, Severns ML. Detection of early hydroxychloroquine retinal toxicity enhanced by ring ratio analysis of multifocal electroretinography. Am J Ophthalmol. 2007;143(5):801-9.e2.

27. Chang WH, Katz BJ, Warner JE, Vitale AT, Creel D, Digre KB. A novel method for screening the multifocal electroretonogram in patients using hydroxychloroquine. Retina. 2008;28(10):1478-86.

28. Tarakcioglu HN, Ozkaya A, Yigit U. Is optical coherence tomography angiography a useful tool in the screening of hydroxychloroquine retinopathy? Int Ophthalmol. 2020;27:1-7.

29. Tsang AC, Ahmadi S, Hamilton J, Gao J, Virgili G, Coupland SG, et al. The diagnostic utility of multifocal electroretinography in detecting chloroquine and hydroxychloroquine retinal toxicity. Am J Ophthalmol. 2019:206:132-9.

30. Goker YS, Ucgul Atılgan C, Tekin K, Kızıltoprak H, Yetkin E, Yesil Karahan N, et al. The validity of optical coherence tomography angiography as a screening test for the early detection of retinal changes in patients with hydroxychloroquine therapy. Curr Eye Res. 2019:44(3):311-5.

31. Ozek D, Onen M, Karaca EE, Omma A, Kemer OE, Coskun C. The optical coherence tomography angiography findings of rheumatoid arthritis patients taking hydroxychloroquine. Eur J Ophthalmol. 2019;29(5):532-7.

32. Ahn SJ, Ryu SJ, Lim HW, Lee BR. Toxic effects of hydroxychloroquine on the choroid: evidence from multimodal imaging. Retina. 2019;39(5):1016-26.

33. Arfeen SA, Bahgat N, Adel N, Eissa M, Khafagy MM. Assessment of superficial and deep retinal vessel density in systemic lupus erythematosus patients using optical coherence tomography angiography. Graefes Arch Clin Exp Ophthalmol. 2020;1:1-8.

34. Telek HH, Yesilirmak N, Sungur G, Ozdemir Y, Yesil NK, Ornek F. Retinal toxicity related to hydroxychloroquine in patients with systemic lupus erythematosus and rheumatoid arthritis. Doc Ophthalmol. 2017;135(3):187-94.

35. Kan E, Yakar K, Demirag MD, Gok M. Macular ganglion cell-inner plexiform layer thickness for detection of early retinal toxicity of hydroxychloroquine. Int Ophthalmol. 2018;38(4):1635-40.

36. Bulut M, Muhammet Kazım E, Toslak D, Akidan M, Başar EK, Cay HF. A new objective parameter in hydroxychloroquine-induced retinal toxicity screening test: macular retinal ganglion cell-inner plexiform layer thickness. Arch Rheumatol. 2018;33(1):52

\section{Publisher's Note}

Springer Nature remains neutral with regard to jurisdictional claims in published maps and institutional affiliations.

Ready to submit your research? Choose BMC and benefit from:

- fast, convenient online submission

- thorough peer review by experienced researchers in your field

- rapid publication on acceptance

- support for research data, including large and complex data types

- gold Open Access which fosters wider collaboration and increased citations

- maximum visibility for your research: over $100 \mathrm{M}$ website views per year

At BMC, research is always in progress.

Learn more biomedcentral.com/submissions 